\title{
Discovery and application of immune biomarkers for haematological malignancies
}

\author{
Dimitrios Zafeiris ${ }^{1}$, Jayakumar Vadakekolathu ${ }^{1}$, Sarah Wagner ${ }^{1}$, \\ Alan Graham Pockley ${ }^{1}$, Graham Roy Ball ${ }^{1}$, Sergio Rutella ${ }^{1}$
}

'John van Geest Cancer Research Centre, College of Science and Technology, Nottingham Trent University, United Kingdom

Corresponding Author:

Sergio Rutella, MD PhD FRCPath

Professor of Cancer Immunotherapy

John van Geest Cancer Research Centre

Nottingham Trent University

Clifton Campus

NG11 8NS

Nottingham, UK

E-mail: sergio.rutella@ntu.ac.uk 


\section{Discovery and application of immune biomarkers for haematological malignancies}

\section{ABSTRACT}

Introduction: Haematological malignancies originate and progress in primary and secondary lymphoid organs, where they establish a uniquely immune-suppressive tumour microenvironment. Although high-throughput transcriptomic and proteomic approaches are being employed to interrogate immune surveillance and escape mechanisms in patients with solid tumours, and to identify actionable targets for immunotherapy, our knowledge of the immunological landscape of haematological malignancies, as well as our understanding of the molecular circuits that underpin the establishment of immune tolerance, is not comprehensive. Areas covered: This article will discuss how multiplexed immunohistochemistry, flow cytometry/mass cytometry, proteomic and genomic techniques can be used to dynamically capture the complexity of tumour-immune interactions. Moreover, the analysis of multidimensional, clinically annotated data sets obtained from public repositories such as Array Express, TCGA and GEO is crucial to identify immune biomarkers, to inform the rational design of immune therapies and to predict clinical benefit in individual patients. We will also highlight how artificial neural network models and alternative methodologies integrating other algorithms can support the identification of key molecular drivers of immune dysfunction.

Expert comment: High-dimensional technologies have the potential to enhance our understanding of immune-cancer interactions and will support clinical decision making and the prediction of therapeutic benefit from immune-based interventions.

\section{Keywords}

Haematological malignancies; leukaemia; lymphoma; multiple myeloma; immunotherapy; biomarker; gene expression profiling; prognosis. 


\section{Introduction}

Tumours are organised tissues that are infiltrated with immune cell populations of both the lymphoid and myeloid lineage [1] and possess both tumour-promoting and tumour-inhibiting properties. Compelling evidence indicates that pre-existing immunological features contribute to the ability of patients with solid tumours to respond to immunotherapy with immunomodulatory agents such as checkpoint inhibitors [2]. The Immune Biomarkers Task Force of the Society for Immunotherapy of Cancer (SITC) recently published recommendations on the discovery of immune-related biomarkers, in which it highlighted the complexity of the tumour microenvironment (TME) and discussed novel tools to analyse the diversity of immune genes, proteins, cells and pathways [3]. A broader understanding of baseline immunity, both in the periphery and in the TME, and of immune escape mechanisms is likely to expedite the identification of biomarkers that are predictive of clinical outcome and elucidate why cancer patients might fail to respond to immunotherapy [4,5]. Powerful technologies such as genome-wide association studies (GWAS), multiplexed immunohistochemistry, high-dimensional blood profiling of immune cells by flow cytometry and mass cytometry are increasingly being integrated in this nascent, but rapidly evolving field. The aim of these approaches is to assess immune competence and the likelihood of patients with solid tumours to respond to immunotherapy. In general, tumour infiltration by leukocyte subsets such as $\mathrm{CD}^{+} \mathrm{T}$ cells and CD45RO+ memory $\mathrm{T}$ cells with specific gene signatures and increased $\mathrm{B}$-cell receptor $(\mathrm{BCR})$ diversity is associated with an improved overall survival (OS), as has been demonstrated by mRNA sequencing data from The Cancer Genome Atlas (TCGA) in 11 solid tumour types encompassing breast, lung, melanoma and lung adenocarcinoma and representing 3,485 patients [6]. In contrast, macrophage signatures predicted poorer survival in most tumour types. The presence of T-cell infiltration contributes to a higher "immunoscore" in patients with colorectal cancer (CRC), which correlates with improved patient prognosis [7].

Whereas the role of anti-tumour immunity in shaping clinical responses to therapy has been thoroughly investigated in melanoma and $\mathrm{CRC}$, our understanding of the role played by individual immune cell types in the control of haematological malignancies remains limited. In principle, haematological malignancies are amenable to immune-mediated therapeutic effects, as suggested by the curative potential of allogeneic haematopoietic stem cell transplantation (HSCT). Although immune checkpoint blockade has only been pursued recently in patients with Hodgkin and non-Hodgkin lymphoma [8,9], the field is expected to advance exponentially, as has already occurred in solid tumour oncology. This will entail a paradigm shift in our current treatment modalities. An imperative for the correct design of clinical trials would be to dissect the determinants of response and resistance to checkpoint 
blockade and to decipher the architecture and composition of the TME, as well as the functional orientation of peripheral blood immune cells in patients with leukaemia, lymphoma and multiple myeloma (MM). Challenges to identifying biomarkers have recently been reviewed [10]. Despite the reciprocal relationship between tumours and the patient's immune system, it is presently unknown whether measurements in blood may correlate with findings from tumour sites, including lymph nodes and bone marrow (BM) [3,11]. In this respect, peripheral blood markers reflecting immune function at baseline ("peripheral immunoscore") have successfully predicted progression-free survival (PFS) in patients receiving vaccines for metastatic breast cancer and prostate cancer [12].

This review will focus on current strategies to interrogate the immunological TME in patients with haematological malignancies, with the objective to subvert cancer-induced immune suppression and identify targets for treatment.

\section{Structure and function of the tumour microenvironment (TME)}

Neoplastic cells activate gene expression programmes in the TME that are supportive of tumour growth and inherently immune suppressive [4]. The TME is increasingly viewed as an attractive candidate for the discovery of predictive and prognostic immune biomarkers $[11,13]$. For instance, intra-tumoural levels of IL-15 strongly correlate with immune cell proliferation and disease recurrence in patients with CRC [14]. An 'immunome' compendium of mRNA transcripts specific for innate and adaptive immune cell populations has characterised the immune composition of the TME in CRC [15]. The patterns of gene expression were remarkably different in patients with significantly prolonged disease-free survival (DFS) and in those with unfavourable outcome. The former showed an over-representation of T-cell-related genes, including $\gamma \delta$ T cells and cytotoxic T cells, macrophages and mast cells. Follicular helper T cells (Tfh) and B cells also exerted a favourable effect on patient outcome. In contrast, patients with poor outcomes showed an over-representation of genes specific for eosinophils, Th2 cells, Th17 cells, Treg cells and NK cells. Interestingly, the in situ immune reaction evolved with tumour progression from stages $\mathrm{T} 1$ to $\mathrm{T} 4$, with most of the $\mathrm{T}$-cell markers decreasing with tumour stage.

Programmed Death Ligand (PDL)-L1 is expressed by cells in the TME, engages PD1 on T cells and triggers inhibitory signalling which prevents T-cell effector function and cytotoxicity [16]. PD-L1 expression in response to cytokine stimuli, most importantly IFN- $\gamma$, has been termed 'adaptive immune resistance' [17]. Co-localisation of inflammatory responses with CD8 and PD-L1 expression has been correlated with improved clinical outcome in patients with metastatic, but not localised, melanoma, implying that 'inflamed' tumours expressing PD-L1 might be more amenable to respond to immunotherapy [17]. A pragmatic classification of solid 
tumours based on their PD-L1 status and presence or absence of tumour-infiltrating lymphocytes (TILs) has been recently proposed [18]. Type I (PD-L1-expressing with TILs) and type II TMEs (PD-L1 negative with no pre-existing TILs) account for approximately $80 \%$ of human melanomas, with type I tumours having the best prognosis [17]. Other tumour types may exhibit a type III TME, in which constitutive PD-L1 expression is driven by oncogenic events rather than adaptive immune resistance, as shown in gliomas with loss of PTEN function [19] and in T-cell lymphomas [20]. Finally, although type IV tumours contain TILs, these show no expression of PD-L1, thereby suggesting a potential role for other immune suppressive circuits in driving immune dysfunction [18].

Intriguingly, three immune profiles have been revealed by clinical studies indicating that patients with 'inflamed' melanomas were more likely to respond to immunotherapy with checkpoint blocking agents $[21,22]$. The immune-inflamed phenotype is characterised by the presence of both $\mathrm{CD}^{+}$and $\mathrm{CD} 8^{+} \mathrm{T}$ cells, often accompanied by myeloid and monocytic cells, and by staining for PD-L1 on TILs and, in some cases, on tumour cells. The immune-excluded phenotype is characterised by tumours in which immune cells are retained in the stroma and fail to migrate and penetrate the tumour itself, and is unlikely to respond to immunotherapy. The third profile, the immune-desert phenotype, is characterised by a paucity of $T$ cells, which is indicative of the absence of pre-existing anti-tumour immune responses, and by the presence of myeloid-derived suppressor cells (MDSCs), M2 macrophages and regulatory T (Treg) cells, which mediate immune suppression or tolerance. The importance of pre-existing, clonally restricted CD8 T-cell responses and of physical proximity between PD1 ${ }^{+}$and PD-L1+ cells in the TME for tumour regression after immunotherapy with PD1 blocking agents has again been demonstrated in patients with metastatic melanoma [23].

In haematological malignancies, the BM represents not only the site of disease initiation and progression, but also a distinctive immunologic microenvironment that contains most developing and mature immune cell types, including long-lived $\mathrm{CD}^{+}{ }^{+}$and $\mathrm{CD} 8^{+} \mathrm{T}$ cells [24]. A recent study identified landmark populations of BM-resident immune cells in mice [25]. Similar cells were grouped into clusters according to their expression of the measured proteins. The scaffold maps allowed the unsupervised visualisation of the immune composition and complexity of murine BMs. In comparison, maps for secondary lymphoid organs exhibited an immune landscape dominated by mature $T$ and $B$ lymphocytes, as well as by myeloid cell clusters mapping closely to the macrophage and dendritic cell (DC) zones. The integration of human mass cytometry data from four healthy donors into the reference map revealed a similar overlay pattern between the two species [25].

In light of their origin from primary and secondary lymphoid tissues, haematological malignancies might be characterised by distinctive mechanisms of immune evasion compared with solid tumours [26]. In principle, haematological malignancies are poorly immunogenic and 
highly immune suppressive. For instance, acute leukaemias disseminate rapidly and constrain protective anti-tumour immune responses through a plethora of immune subversive mechanisms, including the down-regulation of MHC class I and class II expression, the consumption of essential amino acids through arginase-2 (ARG2) [27] and indoleamine 2,3dioxygenase-1 (IDO1) [28], the induction of DC dysfunction, the expansion of Treg cells [29] and the up-regulation of PD-L1 and other negative checkpoint molecules, such as Cytotoxic T-Lymphocyte-associated Antigen-4 (CTLA-4) and Lymphocyte Activation Gene 3 (LAG-3). PD-L1 expression might represent a general strategy of immune evasion among aggressive B-cell lymphomas [30]. The analysis of formalin-fixed, paraffin-embedded (FFPE) tissue biopsies from 237 primary lymphomas has detected PD-L1 protein expression in most nodular sclerosis and mixed cellularity classical Hodgkin's lymphomas $(\mathrm{HL})$, primary mediastinal large B-cell lymphomas, Epstein-Barr Virus (EBV)-positive and EBV-negative post-transplantation lymphoproliferative disorders and EBV-associated diffuse large B-cell lymphomas (DLBCL). This group of neoplasms should then be considered for PD-1/PD-L1-directed therapies, as further discussed below.

Insights into the molecular mechanisms sustaining PD-L1 expression in lymphoma tissues have recently been provided [31]. Conditioned media from T-cell and B-cell lymphoma cell lines were shown to induce PD-L1/PD-L2 expression on macrophages in a Signal Transducer and Activator of Transcription (STAT)-3-dependent manner. In vitro studies pointed to a potential role of lymphoma-derived IL-27B in PD-L1/PD-L2 over-expression, suggesting that an IL-27/STAT-3 axis might be a target for immunotherapy in patients with NHL.

\section{Immune gene signatures}

Innate and adaptive immune responses within the TME can be assessed by gene expression profiling [32]. Immune gene signatures, especially those induced by IFN- $\gamma$, are likely to be powerful biomarkers of response to checkpoint blockade. A considerable body of scientific evidence suggests that tumours responsive to immunotherapies display an inflammatory status which is associated with the concomitant counter-activation of immune suppressive circuits, thereby reflecting immune escape mechanisms. The implication of these observations is that pre-existing immune responses are a pre-requisite for the efficacy of immune checkpoint blockade. For instance, a 10-gene IFN- $\gamma$ score, including genes encoding IDO1, LAG3, PRF1, GZM and other immune-related genes, showed a significant correlation with best overall response (OR) and PFS in patients with advanced melanoma, as well as a nonsignificant association with overall survival (OS) [33].

Importantly, immune-related gene signatures, and not tumour-related gene expression patterns, have been identified as being the main parameters associated with dissemination of 
CRC to distant metastases [34]. Specifically, patients without synchronous metastasis had a significantly increased expression of Th1-related genes, immune cytotoxicity-related genes and MHC class II-related genes compared with patients having metastasis at the time of diagnosis. This study highlights the concept that immune phenotypes, as measured on the basis of multiple parameters, might be a crucial determinant for preventing the metastatic dissemination of tumours to distant sites.

Although immune and genomic landscapes in pre-treatment tumour biopsies correlate with response in patients with melanoma and other solid cancers, robust biomarkers that do not overlap between responders and non-responders have not yet been identified. An interesting study in 53 patients with metastatic melanoma initially treated with CTLA-4 blockade followed by programmed death-1 (PD-1) blockade at the time of progression analysed immune gene signatures in longitudinal biopsies collected at multiple time points during therapy, using a 12marker immunohistochemistry panel and targeted gene expression profiling on a nanoString platform [35]. Adaptive immune gene signatures in tumour samples obtained early during treatment, including the up-regulation of cytolytic markers, $H L A$ molecules, IFN- $\gamma$ pathway effector genes and chemokines, were highly predictive of response to immune checkpoint blockade. Importantly, unique gene expression profiles observed in the TME of patients receiving monotherapy with anti-CTLA-4 or anti-PD-1 antibodies provided insights into the mechanisms of response to distinct forms of immune checkpoint blockade, as well as a compelling rational for the design of combination immunotherapies.

The genomic landscape of tumours has been linked with tumour immunity, with neo-antigens that are predicted by tumour genome meta-analyses being implicated in driving T-cell responses and somatic mutations associated with immunological infiltrates being identified $[36,37]$. A recent analysis of The Cancer Genome Atlas (TCGA) data sets has allowed the identification of correlates of immune cytolytic activity in thousands of TCGA solid tumours [37]. On the basis of transcript levels of two tightly co-expressed cytolytic effector molecules, granzyme A and perforin, differences in cytolytic activities across tumour types were identified, with the highest levels being detected in kidney clear cell carcinoma and cervical cancers. Interestingly, cytolytic activities and expression of IFN-stimulated chemokines (CXCL9, CXCL10 and CXCL11) were associated with the counter-regulatory increase of immune suppressive molecules, including IDO1, IDO2, PDL2 and the C1Q complex, and with a modest, but significant, pan-cancer survival benefit [37].

Finally, immune gene co-expression patterns have been used to identify a subset of highconfidence marker genes in 9,986 solid tumour samples from TCGA [38]. Immune cell scores derived from gene measurements were compared with flow cytometry and IHC data. Cell type scores calculated from a list of 60 marker genes measuring 14 immune cell populations were concordant with flow cytometry and $\mathrm{IHC}$ readings, and allowed comparisons of immune cell 
abundance across different tumour types. Further analyses in an immunotherapy data set (derived from patients receiving anti-CTLA-4 antibodies) showed that cell type gene signatures separated responders from non-responders. Importantly, immune cell scores represent a convenient technique for extracting critical information on the immune contexture of a given tumour in those patients from whom sufficient material for flow cytometry studies is not available [38].

\section{Immune biomarkers in haematological malignancies}

The discovery and validation of immune biomarkers is an area of intense investigation. This section of the article provides examples of individual immune suppressive molecules that could be targeted to improve treatment outcome in patients with leukaemia, lymphoma and MM. We will highlight how on-line tools could expand our predictive capabilities [39] and support the identification of TME immune gene signatures and key molecular drivers implicated in the progression of haematological malignancies, and allow the in-silico validation of experimental findings across multiple data sets (Table 1 and Figure 1) [40-42].

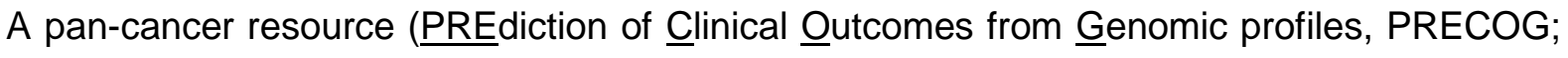
http://precog.stanford.edu) has recently been developed to identify commonalities in prognostic genes from approximately 18,000 human tumours from 166 publicly available cancer data sets with survival outcomes across 39 cancer types, including different types of haematological malignancies [43]. The statistical associations between genes and clinical outcomes were assessed by $z$-scores, which are directly related to $p$ values and represent the number of standard deviations from the mean of a normal distribution. Survival-associated $z$ scores for individual studies were combined to yield meta-z-scores for the prognostic significance of each gene in each cancer type. One of the two clusters identified was associated with inferior clinical outcomes and was functionally linked to cell proliferation [43]. However, proliferation genes were not adversely prognostic in AML. The other large tumour cluster was associated with favourable survival and was enriched in immunological processes and immune-response genes. A new machine-learning tool, known as CIBERSORT [41], was subsequently applied to PRECOG data to comprehensively map compositional differences in tumour-infiltrating leukocytes in relation to patient outcome. Expression profiles for 22 distinct leukocyte subsets were used as input.

CIBERSORT revealed remarkable differences in relative leukocyte composition between haematopoietic and solid tumours. As shown in Figure 2, CIBERSORT inferred high frequencies of plasma cells in MM specimens and the predominance of B-cell signatures in B-cell malignancies, thereby underpinning its utility for identifying the cell of origin (COO) in diverse tumour types [43]. Pooling cancer types allowed the identification of global leukocyte 
prognostic patterns. Higher frequencies of estimated T-cells, especially intra-tumour $\gamma \delta \mathrm{T}$ cells, correlated with superior survival. In contrast, infiltration with polymorphonuclear cell fractions was the most significant adverse prognostic factor. Finally, signatures of polarised M2 macrophages predicted worse clinical outcome than pro-inflammatory M1 macrophages.

Acute myeloid leukaemia. Immune responses are defective in patients with AML due to the presence of powerful immune suppressive circuits that are activated by soluble factors and immune checkpoint molecules, including PD-L1, TIM-3 and IDO1 [28,44]. Serum kynurenine and tryptophan levels at diagnosis, a measure of systemic IDO1 activity, correlate with patient outcome [45]. Testing of checkpoint blockade is currently being pursued in patients with AML (www.clinicaltrials.gov, NCT02892318; NCT02508870; NCT02532231; NCT02771197; NCT03065400; and NCT03066648). Although the mutational burden and immunogenicity of AML are inherently low, immunotherapies boosting T-cell functions might be effective, especially in the setting of minimal residual disease, and particularly when combined with checkpoint inhibition or other strategies to overcome leukaemia-induced immune dysfunction. Importantly, genetic mutations such as $t(8 ; 21)$ and inv(16) directly affect the expression of CD200 (a suppressor of macrophage and NK cell function) and CD48 (the ligand for the activating NK receptor CD244), respectively.

Chronic lymphocytic leukaemia. Chronic lymphocytic leukemia (CLL) is characterized by profound immune defects that are already present in the early stages of the disease and these lead to a heightened vulnerability to severe infections. The frequency of PD-L1-expressing monocytic MDSCs might be significantly increased in untreated CLL patients compared with healthy controls [46]. MDSCs from patients with CLL have been shown to modulate T-cell function in vitro and to induce Treg cell differentiation, partly through their expression of IDO1. Plasmacytoid DCs, which play an undisputed role in anti-viral immunity as well as antileukaemia responses, are reduced in number and function in patients with CLL as a result of decreased expression of FMS-like tyrosine kinase 3 receptor (Flt3) and Toll-like receptor 9 (TLR9) [47]. These represent molecular targets for restoring immune competency. Functional screening assays have identified multiple inhibitory ligands in CLL which impair actin synapse formation in T cells, including CD200, CD270, CD274, and CD276 [48]. Importantly, lenalidomide, an immune-modulatory drug, can down-regulate tumour cell inhibitory molecule expression, thus preventing the induction of T-cell defects. Blockade of the PD1 pathway with pembrolizumab has been successfully pursued in patients with CLL and Richter transformation into DLBCL [49]. Objective responses were documented in four out of nine patients with Richter transformation and in 0 out of 16 patients with relapsed CLL. Analyses of pre-treatment tumour specimens showed increased expression of PD-L1 and a trend 
towards increased expression of PD1 in the TME of patients with confirmed clinical responses. All responding patients with Richter transformation had received prior therapy with ibrutinib, a Bruton's tyrosine kinase inhibitor.

Chronic myeloid leukaemia. Targeted treatment with tyrosine kinase inhibitors (TKIs) has revolutionised the fate of patients with chronic myeloid leukaemia (CML). Intriguingly, TKIs exert a variety of off-target immunological effects (comprehensively reviewed in ref. [50]), suggesting that novel combinations of molecularly targeted agents and immunotherapies may further improve clinical success rates for CML. Mass cytometry has enabled the identification of prognostic immune biomarkers in longitudinally collected samples from patients with $\mathrm{CML}$ receiving tyrosine-kinase inhibitors (TKIs) [51]. An increase of circulating CD8 ${ }^{+}$cytotoxic $T$ cells occurred after 7 days of TKI therapy and, importantly, changes in single-cell transduction events, including down-regulation of phosphorylated CREB S133 and up-regulation of phosphorylated STAT3, reflected molecular response at 3 and 6 months.

Hodgkin's lymphoma (HL). Classical HL is characterized by a paucity of malignant Hodgkin and Reed-Sternberg (HRS) cells in lymphoid tissues, accompanied by a massive infiltrate of reactive cells, including leukocytes and stromal cell types. Modulators of innate and adaptive immune responses such as galectin-1 (Gal-1), a member of a highly conserved family of carbohydrate-binding proteins, are over-expressed by Reed-Sternberg cells, thereby leading to depletion of Th1, Th17 and cytotoxic T cells, with an expansion of Treg cells in the TME [52]. Gal-1 levels are elevated in patient serum in association with clinical parameters such as Ann Arbor stage, areas of nodal involvement and International Prognostic Score. In classical HL, tumour-associated macrophage (TAM) and monocyte signatures in diagnostic FFPE lymph node specimens have been associated with high risk of primary treatment failure and with decreased PFS and OS [53,54]. Among the 27 individual genes with a discriminative power for outcome prediction exceeding that of the best clinical variable (patient age), matrix metallopeptidase-1 (MMP1) was over-expressed in patients with treatment failure.

Non-Hodgkin's lymphoma (NHL). NHLs are typically associated with chronic inflammatory and autoimmune conditions, with severe immune dysregulation being an established risk factor and a hallmark of the disease. For instance, high pre-treatment plasma levels of CXCL13, IL6 and IL-10 predict worse PFS and OS in patients with AIDS-related NHL (AIDS-NHL) receiving intensive multi-agent chemotherapy and immunotherapy with rituximab [55]. Longitudinal monitoring of cytokine levels 1 to 5 years preceding NHL diagnosis has identified cytokines and other molecules associated with chronic immune activation, such as IL-6, IL-10 and TNF- $\alpha$, as predictors of the development of systemic AIDS-NHL [56,57]. Similarly, circulating levels of B-cell attracting chemokine 1 (BCA-1), soluble TNF receptor 2 and soluble vascular endothelial growth factor (VEGF) 2 have been correlated with the risk of NHL in 
advance of diagnosis [58]. Similarly, genetic variants of toll-like receptor (TLR)-9 which lead to increased transcriptional activity in mononuclear cells might increase NHL susceptibility [59]. Finally, three independent population-based case-control studies have revealed a correlation between $\mathrm{NHL}$ risk and single-nucleotide polymorphisms within 12 innate immunity genes, including IL-1 receptor antagonist and IgG Fc receptor $2 \mathrm{~A}[60]$.

Follicular lymphoma (FL). FL is the second most common type of NHL, accounting for approximately $20 \%$ of all cases. The malignant B cells in FL are of germinal-centre origin. $\mathrm{FL}$ is clinically heterogeneous, with some patients experiencing an indolent clinical course and others having rapidly progressive disease. A multivariate model of survival was constructed using whole-genome microarray data from lymph node tissues from 191 patients with untreated FL [61]. This study identified two distinct immune response gene signatures, immune-response 1 and immune-response 2, which reflected the biological characteristics of the non-malignant immune cells within the biopsy specimens and were molecular predictors of the length of survival in patients with FL. The immune-response 1 signature included genes associated with $\mathrm{T}$ cells and genes which were highly expressed in macrophages. Genes in the immune-response 2 signature were preferentially expressed in macrophages and DCs. Importantly, the gene expression-based model predicted patient survival independently of clinical variables such as the International Prognostic Index (IPI) and the presence or absence of B symptoms [61].

Other immune cell types, such as tumour-associated mast cells and tumour-associated macrophages, have prognostic importance in FL. Mast cell infiltration was detected using immunohistochemistry and was shown to negatively affect progression-free survival in patients with FL receiving a combination of immunotherapy (rituximab) and chemotherapy (CHOP) [62]. The prognostic impact of mast cell infiltration was again independent of the FL IPI. The mechanisms by which mast cells reduce the efficacy of antibody-based therapies in FL remain to be determined and might include the negative regulation of macrophage activity and antibody-dependent cellular cytotoxicity through the expression of Fc $\gamma$ receptors which can engage rituximab [62].

Diffuse large B-cell lymphoma (DLBCL). DLBCL is the most common subtype of NHL, representing more than $30 \%$ of all adult $\mathrm{NHL}$ cases diagnosed in Western countries, and is characterised by an aggressive clinical course. In spite of improved response and survival rates after the addition of rituximab to the therapeutic armamentarium, up to $40 \%$ of patients with DLBCL experience relapse and have a poor prognosis.

Gene expression profiling and next-generation sequencing have been instrumental to the identification of molecular subtypes of DLBCL, which are not obviously related to histological subtypes of DLBCL and are associated with a remarkable divergence in clinical behaviour. 
Patients with activated B-cell-like ( $A B C)$ gene signatures have a shorter survival compared with patients with the other two molecular subtypes, i.e., germinal centre B-cell (GCB) and primary mediastinal B-cell lymphoma signatures [63]. Non-GCB type DLBCLs are enriched with PD-L1-expressing tumours and might benefit from targeted immunotherapies [64].

DLBCLs have a heterogeneous immune infiltrate, which includes macrophages, DCs, NK cells, T-cell subsets and B cells. Interestingly, pre-treatment gene expression of CD68 as well as immunohistochemically-defined $\mathrm{CD} 8^{+}$macrophages might correlate with better outcome in patients with DLBCL receiving chemo-immunotherapy, independently of IPI scores or molecular subgroups [65]. In contrast, macrophage infiltration was negatively correlated with OS in patients treated without rituximab, leading to the hypothesis that rituximab administration might switch macrophage profile towards a tumour-promoting phenotype.

Tissue microarray immunohistochemistry with automated scoring of FoxP3, CD68 and microvessel (CD34) density (MVD) has been shown to stratify patients with DLBCL into risk groups and to predict prognosis [66]. Patients in the high-risk group had significantly worse EFS and PFS, suggesting that TME components should be considered as an important tool to predict patient survival. The NanoString digital hybridization approach for RNA quantification has been employed to detect immune effector and checkpoint genes in FFPE biopsies from patients with DLBCL [67]. The product of the immune effectors $(C D 4 \times C D 8)$ in a ratio with the product of checkpoints (PD-L1×M2 macrophages) was used to identify low-immune and highimmune groupings of patients with significant differences in 4-year survival. Patients with a GCB or an ABC molecular subtype of DLBCL and a high immune ratio had a significantly extended survival compared with GCB and $A B C$ patients with a low immune ratio, suggesting that the balance of anti-tumoural immunity, i.e., the ratio of immune effector cells to negative checkpoint molecules, might have an important prognostic value in DLBCL.

Primary mediastinal large B-cell lymphoma (PMLBCL). PMLBCL, a distinct and uncommon subtype of DLBCL, is more frequent in young females and originates in the mediastinum, presenting with features of local invasion [68]. Aberrations consisting of structural genomic rearrangements, missense, nonsense, and frame-shift mutations involving the major histocompatibility complex (MHC) class II trans-activator CIITA have been detected in approximately $50 \%$ of patients with PMLBCL [69]. Genomic lesions in CIITA resulted in decreased protein expression and reduction of MHC class II surface expression, favouring the establishment of an immune-privileged microenvironment in PMLBCL.

PMLBCL has a unique transcriptomic signature which is close to classical $H L$ and is characterised by constitutive expression of PD-L1 and PD-L2. Amplification and/or translocations involving chromosome 9p24.1, a region that includes PDCD1LG2-encoding PD-L2, are a common event in PMLBCL but not in DLBCL [70]. This observation entails that 
PMLBCLs might be susceptible to PD1 blockade. A recent clinical trial run as part of the KEYNOTE-013 multi-centre phase $1 \mathrm{~b}$ study has shown decreases in target lesion in approximately $80 \%$ of patients evaluable by imaging [71]. Overall, median survival was not reached for treated patients. Drug-related adverse effects were observed in $60 \%$ of the patients and were manageable. Other immune suppressive circuits in patients with PMLBCL include the down-regulation of HLA-DR expression and the decrease of cytotoxic CD8 ${ }^{+} \mathrm{TIA} 1^{+}$ T cells, features which correlate with shorter progression-free survival [72].

Multiple myeloma (MM). Patients with MM suffer from severe and complex defects of humoral and cellular immunity, including an increased production of immune suppressive cytokines [73] and an expansion of immune regulatory cell types [74]. IL-17, IL-21, IL-22 and IL-23, and Th17 cells are increased in patients with MM compared with healthy donors [75]. In particular, IL-17 might promote MM growth, colony formation and development in a murine xenograft model.

PD1 and its ligands are broadly expressed in the TME of MM, in which they may mediate immune evasion mechanisms [76]. Similarly, PD-L1 expression, as well as IDO1 function, are increased in patients with MM compared with healthy controls [77,78]. Of interest, PD-1/PDL1 blockade may abrogate bone marrow stromal cell (BMSC)-induced MM growth, an effect which is further potentiated by lenalidomide and correlates with the induction of intracellular expression of IFN- $\gamma$ and granzyme B in effector cells. BMSCs from patients with MM also inhibit the lysis of MM cells in a cell contact-dependent fashion by inducing the expression or surviving, a caspase-3 inhibitor, and down-regulating CD95 expression [79].

A thorough characterisation of T-cell, DC and NK cell phenotypes has demonstrated a decreased expression of T-cell activation markers, Th1 cells and proliferation markers in patients with high-risk 'smouldering' MM compared with healthy controls [80]. The fact that treatment with the immune modulating drug lenalidomide translated into an increase of functionally active T-cells, even when combined with low-dose dexamethasone, suggests that immune modulatory drugs might delay the progression of smouldering $\mathrm{MM}$ to overt $\mathrm{MM}$.

Finally, MM can avoid immune surveillance via the transfer of membrane proteins in a process known as trogocytosis [81]. For instance, CD86 and HLA-G from malignant plasma cells can be acquired by T-cells residing in the BM compartment. HLA-G-expressing T-cells exhibited a regulatory potency similar to that of natural Treg cells. Interestingly, the association of CD86 or HLA-G expression with a poor prognosis suggests the induction of in vivo immune suppression.

\section{Future immunotherapy approaches for haematological malignancies}


T-cell engineering with synthetic chimeric antigen receptors (CAR) is revolutionising current treatment paradigms for patients with B-cell malignancies. Durable clinical responses up to 24 months were induced by CD19-directed CAR T cells in $90 \%$ of children and adults with relapsed or refractory B-cell acute lymphoblastic leukaemia (ALL) [82]. Remissions caused by CD19-specific CAR T cells were correlated with high serum levels of IL-15 in patients with lymphoma [83]. CD30-specific CAR T cells have been safely and successfully administered to patients with HL [84]. Clinical responses to CAR T cells could be improved by targeting tumour-induced immune suppression with pembrolizumab [85] or by antagonising IDO1 activity with lymphodepleting drugs such as fludarabine and cyclophosphamide [86]. Innovative approaches are currently being developed to target T-cell malignancies with CD7specific CAR T cells [87] and to eradicate antigen-loss relapses of myeloid malignancies with dual CD19-CD123-redirected CAR T cells [88]. Anti-myeloma activity of CAR T cells specific for B-cell maturation antigen (BCMA) has recently been shown in one patient with chemotherapy-resistant disease [89]. Intriguingly, clinical responses have been achieved using CD19-specific CAR T cells in one patient with MM despite the absence of CD19 expression on malignant plasma cells [90]. Finally, a phase I clinical trial in 16 patients with relapsed or refractory $\mathrm{B}$-cell malignancies $(\mathrm{MM}, \mathrm{NHL}$ and $\mathrm{CLL}$ ) has shown complete clinical responses after the infusion of CAR T cells specific for malignancy-associated $\kappa$ light chains [91].

Bi-specific antibody construct are also being implemented in patients with advanced acute leukaemia and with NHLs. Treatment with blinatumomab, a CD3-CD19 bi-specific T-cell engager antibody, has resulted in significantly longer median OS than chemotherapy (7.7 months versus 4.0 months) in a randomised clinical trial in adults with relapsed or refractory ALL [92]. Blinatumomab induces the expansion of both naïve and memory CD4 ${ }^{+}$and CD8 ${ }^{+} \mathrm{T}$ cells in patients and might skew T-cell receptor repertoires [93]. Immune biomarkers which predict clinical responses to blinatumomab have not been identified yet. Interestingly, PD-L1 expression levels may be higher in children with ALL refractory to blinatumomab [94].

Evidence from clinical trials in patients with solid tumours suggests that combination strategies that synergise with immune checkpoint blockade might be more effective than single-agent immunotherapy, as reviewed elsewhere [95]. It is anticipated that the rational development of personalised combination immunotherapy approaches for patients with haematological malignancies will be informed by the discovery and validation of immune biomarkers.

\section{Multiplexed tissue biomarker imaging}

The direct assessment of immune phenotypes and their spatial relationship by multiplexed techniques provides essential information which is highly complementary to gene expression 
profiling and may allow the discovery of composite predictive biomarkers [32].

Multiplexed immunofluorescence allows the detection of up to 30 proteins in regions of interest within the TME. Multiple fluorophores can be applied on a single tissue section and are interrogated using a multi-spectral microscope [11,96]. This technology enables a comprehensive characterization of the topography and spatial relationship between tumour cells and microenvironmental cell types, including immune cells. Of relevance, the density of $\mathrm{CD}^{+} \mathrm{T}$-cell infiltrates in the invasive margins of melanoma lesions has been associated with expression of the PD1/PD-L1 immune inhibitory axis and with clinical responses to anti-PD-1 immunotherapy [23]. Quantitative image analysis could also be valuable in dissecting the spatial distribution of DCs at different maturation stages within the tumour-draining lymph nodes, thus providing insights into actionable circuits of immune dysfunction [97].

NanoString Technologies (Seattle, USA) has recently developed a multiplexed immune profiling approach to measure the expression of up to 800 targets at protein and RNA level on a single FFPE tissue slide [98]. This Digital Spatial Profiling platform allows the analysis of tumour geography and the delivery of digital counts of biomarker expression with single-cell resolution. It is expected that multiplexed technologies can be applied to the investigation of immune cell distribution in tissue biopsies from patients with haematological malignancies. However, the extensive data that are generated with the use of the above technologies will need to be integrated and 'converted' into useful information using novel bioinformatics approaches.

\section{Machine learning}

Advances in bioinformatics have led to a vast amount of data being generated at an accelerated pace. Next generation RNA and DNA sequencing methods is providing access to incredibly detailed information on entire genomes and allowing us to interrogate more potential biomarkers with an increased level of accuracy. This massive volume of data creates a problem of complexity which makes it impossible to use traditional methodologies.

Machine learning is an interdisciplinary field of bioinformatics which employs a data-driven class of algorithms to find solutions to a given problem by studying, for example, gene expression patterns across many cases / patients. Although widely and successfully used in biology and biomarker discovery studies, the use of these approaches in haematological malignancy studies has, to date, been extremely limited.

Many approaches have been developed, each of which will be explained in terms of their utility here. These approaches can be broadly characterised in two distinct groups; supervised and unsupervised machine learning. 


\subsection{Supervised learning.}

Supervised learning approaches are widely applied and use source features to predict a target class [99]. The supervised approach allows the algorithm to train itself by detecting patterns in large datasets that are predictive of the target class, for example, how does IFNG behave in acute myeloid leukaemia compared to acute lymphoblastic leukaemia? We can make use of previous studies and adjust the algorithm parameters so that it accounts for this information. One major advantage is that such approaches are tolerant of the highly complex, non-linear and noisy data that are often found in biological systems.

Artificial Neural Networks. Artificial Neural Networks (ANN) are statistical models emulating the function of a network of human neurones for the purposes of encapsulating information in order to analyse large, complex datasets. The learning process is based on the mathematical interconnections between the processing elements that constitute the network architecture [100]. This allows them to classify cases based on data by assigning a numerical weight value to each input and adjusting them as they sample the data, effectively learning the optimal solution. The main advantages of ANNs include their high fault and failure tolerance, scalability and consistent generalisation ability, all of which allow them to effectively predict or classify new, fuzzy and unlearned data [100,101]. Additionally, they have been recently used to create panels of biomarkers that, when used in conjunction with each other, predict breast cancer [102].

The original ANN architecture, as proposed by Rosenblatt in 1958, was based on the concept of a single artificial processing neuron with an activation threshold, adjustable weights and bias. However, this could only be used for the classification of linearly separable patterns, as it only learns when an error occurs during testing. This is rarely the case with complex problems such as cancer, as patients do not typically fall into a standard distribution and variance in the data is often significant. Typically, ANNs make use of a Multi-Layer Perceptron (MLP) which is made up of multiple perceptrons arranged in layers of three or more, consisting of input, hidden and output layers. These consider the predictor variables, perform feature detection through an activation function and output the results of the algorithm respectively.

ANNs have been successfully used to predict and classify data in different contexts, such as early detection [103], prediction of long-term survival [104] and biomarker discovery in breast cancer [102,105], classification of colorectal cancer tissues [106] and discrimination between benign and malignant endothelial lesions [107]. One of the major disadvantages of ANNs is their liability to overfit when the parameters have not been optimised. Moreover, they often receive criticism for their "black box" approach which allows for little to no interpretation of the results and process. 
Support Vector Machines. Support Vector Machines (SVM) are supervised classification and regression algorithms that are primarily designed to solve binary problems. They are focussed on finding a hyperplane which separates two classes [108] and have been successfully used in pattern recognition and classification. The popularity of SVMs is a result of the availability of a large variety of kernels (functions that separate data) which can be broadly split into linear, polynomial, sigmoid and radial basis function categories. The greatest advantage of SVMs when compared to similar machine learning methods, is that selecting the correct kernel function enables the analysis of non-linear data, and overcomes the curse of dimensionality. However, the introduction of more features increases the complexity, and therefore the computing power required. Notwithstanding the practical issues, SVMs have been used for analysing high density data, such as RNA, miRNA and proteomics, and they remain one of the most popular classification methods, especially for cancer prediction and prognosis [109112].

As indicated above, disadvantages of SVMs include the computational processing power and the time, although much like ANNs, these problems are quickly being addressed. A more crucial issue facing the application of SVMs is choosing the appropriate parameters and kernel that will allow for sufficient generalization because of the high algorithmic complexity which is required for 'real' data. As a result, the use of SVMs is less supported in settings which require interpretation and decision-making [108].

Decision Trees and Random Forests. Tree based methods involve stratifying a dataset into multiple categories (similar to hierarchical clustering) that can then be used to predict possible outcomes based on the values of the input variables. These methods can be used for both classification and regression problems. Decision tree classification algorithms pose a series of questions based of the features of the dataset and train to split those features into separate categories, thereby resulting in a dendrogram.

Although the advantages of these methods are that they are computationally efficient, have good predictive values and their results are easy to interpret, their predictive accuracy tends to be lower than their counterparts. To mitigate this issue, methods such as random forests, bagging and boosting are used to construct multiple trees in parallel. These can then be combined to provide a significant boost to their prediction accuracy at the cost of some of their interpretability.

Bayesian Networks. Bayes theory states that the conditional probability of A given $B$ is the conditional probability of $B$ given $A$ scaled by the relative probability of $A$ compared to $B$. Using Bayesian networks, the association between a set of variables or nodes can be determined through joint conditional probability distributions [113]. 
Although such approaches have been used for multiple biological applications such as inferring cellular networks, modelling protein signalling pathways, data integration, genetic data analysis and classification [114-116], they are limited by the fact that they need larger than average datasets to obtain sufficient prior probabilities to produce an accurate outcome. This in turn makes them extremely computationally expensive. Moreover, they tend to perform poorly on high dimensional data and their output tends to be complex and as such, can be hard to interpret for non-specialists. Finally, it should be noted that Bayesian networks are not truly Bayesian in nature. They simply adhere to the basic rules of Bayesian statistics on probabilistic inference. It would be more accurate to say that Bayesian networks are directed graphical models with Bayesian elements.

6.2 Unsupervised Learning. Unsupervised machine learning approaches are used when the desirable or predefined output is not available. The goal of unsupervised learning problems is to discover the structure of the data and define groups of similar examples, commonly called clustering. Clustering is one of the main unsupervised approaches and it functions by assigning data points to natural categorical classes or groups, based on similarity or difference of patterns without prior training [117].

Unsupervised learning approaches are best used when the subject is a very large dataset with few known variables. This allows the user to find natural patterns in the data and discover novel groups that have not been previously established and using which training can be undertaken. They have been most commonly used to distinguish patterns in microarray data by clustering genes based on their expression levels [118-120].

Hierarchical Clustering. Hierarchical clustering, the most common unsupervised learning technique, has been widely used for the analysis of microarray data. It is based on measuring distances between data points and defining the first instance of each point as a single cluster, followed by merging the clusters according to distance, with smaller distances between clusters indicating greater similarity. The process continues in an iterative manner until all samples have been used to produce a phylogenetic tree-like structure of the clusters (dendrogram), with individual samples at the bottom, and a cluster containing every element in the dataset at the top [117]. Some of the most popular methods to determine cluster hierarchy include Single-linkage, Complete-linkage, Average-linkage and Centroid distance. The major limitation of the hierarchical clustering approach is that as the clusters grow, they might not be representative of the objects within, and it is hard to rectify mistakes that occur early in the clustering process. 
K-means Clustering. Much like hierarchical clustering, K-means clustering is a partition algorithm which works by arbitrarily grouping objects into a predetermined number of clusters in an iterative manner. The centroid-average expression of each cluster is assigned randomly, based on the Euclidean distance between each object and the closest cluster average. The algorithm then recalculates the average centroid expression, based on the mean of all objects assigned to it, and repeats the process until convergence is reached, where the average expression of each cluster does not change significantly [117]. Unlike hierarchical clustering, this method has the advantage of being able to deal with large datasets and as a result has been applied to more complex problems. However, the major drawback of this method is that repeating the test can produce significantly different results, as the final assignment of clusters is dependent on the initial random assignment of objects [121].

Principle Component Analysis. Reduction in dimensionality is often necessary for a visual inspection of high-dimensional data, as the number of variables being investigated often exceed the number of samples. This leads to data points being scarcely distributed in a high dimensional feature space [122]. The aim of Principle Component Analysis (PCA) is to map the original data into its principle components by linearly transforming the data to reduce dimensionality. These principle components are orthogonally arranged, mutually uncorrelated linear combinations of the original variables and are often ranked by the amount of variance they can explain in the data. The highest ranked components contain most of the relevant information, whereas low ranked principle components can be removed if they are not required. This approach is often used as a visualisation tool and pre-processing step for classification and clustering [117].

6.3 Novel Approaches. Two bioinformatics approaches developed recently have managed to provide novel solutions to common problems related to big data analysis.

CIBERSORT. CIBERSORT is a platform for characterising the cell composition of tissues based on their gene expression profiles [41]. Traditionally, immunohistochemistry and flow cytometry have been used to answer such questions and, although highly successful, they are limited by their reliance on known markers as well as the fact that these techniques are harmful to cells, likely altering the results. CIBERSORT manages to achieve similar results to these techniques using the RNA mixtures of the desired tissue. It is an SVM regression algorithm which allows the user to differentiate cell types in large datasets. CIBERSORT has been proven to have superior performance and be substantially more accurate over traditional Machine Leaning methods when the samples studied were unknown, noisy or closely related. However, limitations include its reliance on a reference database, the fidelity and size of which 
are considerable factors in the algorithm's ability to classify the cell samples, the lack of a $p$ values for detection limits and a systematic over- and under-estimation of certain cell types. Much like all major machine learning approaches, these problems are being mitigated as more computing power becomes available and the size and fidelity of databases increases.

Hive Plots. One of the key challenges in the field of bioinformatics is the issue of visualisation. Although the approaches discussed previously have expanded the field of biomarker discovery by allowing researchers to consider new possibilities, their use in diagnostics is limited by the fact that the results often require expert specialists to interpret. If these approaches are to achieve widespread use by clinicians for prognosis, it is paramount to have a clear and easily understandable output. Developed by Krzywinski et al. [123], hive plots offer an alternative network visualisation method to traditional maps. These maps, usually produced by software such as Cytoscape, Gephi, Netminer and more recently, programming languages such as $\mathrm{R}$, have a tendency to include an overwhelming amount of information, leading to networks that need to be analysed with sorting algorithms to be readable and hard to interpret. Moreover, complexity increases exponentially as more information is included. Hive plots offer a rational visualisation technique which groups nodes based on specific properties determined by the user. The properties can be inherent network statistics, or information such as features of clinical data.

\section{Expert commentary}

A patient's immunological profile should be considered a highly dynamic framework, which is affected by variations in tumour genetics, epigenetics and micro-RNA expression, age, microbiome composition, pharmacological agents and environmental factors including infections and exposure to sunlight [21]. There is an emerging need to identify immune biomarkers of cancer response to immunotherapies [39]. High-dimensional technologies will also enhance our understanding of TME-cancer interactions and will support the prediction of therapeutic benefit from immune-based interventions (Figure 3). Immune assays for biomarker discovery, as well as sample collection and handling, must be harmonised and standardised for investigators to be able to compare and share results [3].

Although the role of immune gene signatures in stratifying patients with haematological malignancies and in supporting clinical decision making remains to investigated, efforts are being devoted to the discovery of prognostic signatures (to predict outcome independent of therapy), predictive signatures (to assist in treatment selection according to therapeutic effectiveness) and mechanistic immune signatures in patients with solid tumours [124,125]. Prognostic signatures help predict outcome independent of therapy, whereas predictive 
biomarkers and signatures (before treatment) might assist in treatment selection according to therapeutic effectiveness. Mechanistic signatures should capture the maximal intensity of immune responses which occur in tumour lesions that are about to regress after immunotherapy administration [124]. Importantly, comprehensive analyses have indicated that prognostic, predictive and mechanistic immune signatures across different immunotherapeutic strategies might overlap qualitatively and converge into a common pathway [124]. It is becoming evident that solid tumours which are responsive to treatment generally have an inflammatory status, indicative of pre-existing immune responses, as well as expression of cytolytic markers with concomitant counter-activation of immune suppressive and immune escape circuits, which should be targeted with rational combinatorial approaches (for instance, PD-L1 blockade coupled with small-molecule IDO1 inhibitors [126]).

Because of inherent limitations of gene expression profiles, other approaches, such as flow cytometry, quantitative immunohistochemistry and next-generation sequencing for T-cell antigen receptors or similar technologies (multi-N-plex quantitative PCR, spectratyping and immune phenotyping) are recommended to thoroughly characterise the immunological landscape of the TME and to establish predictive models [23], as recently reviewed by the Immune Biomarkers Task Force of the Society for Immunotherapy of Cancer [11]. Conceivably, the analysis of multi-dimensional data sets will be instrumental to mapping the immunological landscape of haematological malignancies, to revealing potential immune biomarkers and informing the rational design of immune therapies. A combination of personalised transcriptomic and proteomic measurements will likely be required to develop accurate immune gene signatures in individual patients (Figure 4). The collection of comprehensive immunological profiles or 'cancer-immune set points' will inform personalised clinical trials and support the prediction of anti-cancer responses to immunotherapy [21].

\section{Five-year view}

Immune profiling of patients with haematological malignancies is expected to underpin the discovery and validation of new biomarkers, and to foster the clinical implementation of a more refined and personalised approach to immune-based interventions. Immune parameters could be used to build dynamic frameworks and to support treatment allocation to cancer patients, such as the recently proposed 'cancer immunogram' [2], the aim of which would be to visualise the state of cancer-immune interactions in individual patients with cancer and to discuss treatment options in a personalised manner. The information required to build a cancer immunogram should include tumour foreignness, patients' immunological status, evidence for tumour infiltration with T cells, expression of checkpoints and other molecules inhibiting T-cell function, and tumour cell sensitivity to immune effectors, including the inactivation of antigen 
processing machinery components [2]. The above parameters should be collected from the blood and/or tumour tissues using transcriptomic approaches, high-resolution immune phenotyping, spatially-resolved immunohistochemistry and standard immunological assays [11].

Strategies that combine different methods of capturing the immunological status of the TME may particularly support the development of composite predictive biomarkers for immune checkpoint inhibition in the Haematology clinic, an area that is expected to flourish during the next few years [32]. For example, gene expression profiling approaches, such as nanoString Technologies' digital platform [98], coupled with multiplexed immunohistochemistry techniques, will allow investigators to quantify mRNA species and multiple proteins expressed in cell populations within morphologically defined regions of interest in the TME, thus providing crucial information about the topography and spatial localisation of immune cells at different tumour stages or after treatment with immunotherapies.

Finally, new bioinformatics approaches are being developed to unravel the complexity and multi-dimensionality of datasets obtained through transcriptomic, sequencing and proteomic techniques, to identify responders and non-responders and to stratify and select patients based on immune gene signatures in the TME [127]. In the foreseeable future, immune biomarkers might guide the development and personalisation of combination immunotherapy approaches [10]. As machine learning is becoming an integral part of biomarker discovery, it presents its own set of challenges with the first one being the constant need for higher computational power. As the size of the available datasets and the complexity of the platform technologies (e.g. the move to 1million SNIP probes on a chip, or the advent of RNA deepSeq. studies) increases, computational requirements will increase exponentially. While current advances in GPU-accelerated parallel computing, solid-state drives and the availability of highly parallel cloud computing solutions have allowed for a significant increase in processing power, it is proving insufficient to handle some of the more complex questions. There is also a trend occurring where the processing power increases so the analyses that are conducted become deeper and more detailed.

The quality and size of the datasets is a key factor in ensuring high quality results. Not only have the standards for size been raised, with datasets like METABRIC and databases like TCGA, TARGET, ADNI and others providing access to data from thousands of cases, but the quality desired in such data is going to keep increasing as well. This is compounded by the fact that as more data becomes publically available it can be used to validate tests results with ever-increasing accuracy. If comparative analysis is conducted across multiple cancers of different tissue origin (so called pan cancer studies) or between the ever-increasing number of molecular subtypes of given cancers a greater need for processing will be required.

Finally, further research is required in the more recent areas of machine learning, primary 
among them being network inference studies and the so called deep learning and deep mining strategies. Understanding how questions of interest interact and affect each other, such as how genes regulate each other in a given disease, and use machine learning to model more possibilities than could be reasonably studied manually [75] will further increase the potential venues of research.

\section{Key issues}

- Identification of predictive/prognostic immune biomarkers in the blood and TME of patients with haematological malignancies

- Development of prognostic and mechanistic immune gene signatures in patients with haematological malignancies receiving immunotherapies, including checkpoint blockade

- Handling and analysis of multi-dimensional data sets using artificial neural network models

- Prospective validation and incorporation of immunological parameters into personalised routine clinical practice (patient stratification, treatment allocation)

\section{Financial disclosure/Acknowledgements.}

The Authors have no financial involvement to disclose.

The Authors' work is supported through research grants from the Roger Counter Foundation (Dorset, UK), the Qatar National Research Fund (NPRP8-2297-3-494) and the John and Lucille van Geest Foundation. 
Figure and Table Legends.

Figure 1: Identification of potential immune biomarkers using publicly-available on-line tools. Blood-Spot (http://servers.binf.ku.dk/bloodspot/) provides plots of gene expression in normal and malignant haematopoietic cells at different maturation stages based on curated microarray data [128]. We selected MX1 (Myxovirus [Influenza] Resistance 1), an interferon (IFN)-inducible gene, as an example of use of Blood-Spot to interrogate human AML data sets. Panel A: mRNA expression levels are depicted across a broad range of normal haematopoietic differentiation stages (first 11 columns on the left; data derived from Gene Expression Omnibus Series GSE42519) and in patients with different cytogenetic subgroups of AML (data derived from Gene Expression Omnibus Series GSE13159, GSE15434, GSE61804, GSE14468, and from The Cancer Genome Atlas [TCGA]). HSC = haematopoietic stem cell; MPP = multi-potential progenitor; $\mathrm{CMP}=$ common myeloid progenitor; GMP = granulocyte-monocyte progenitor; $\mathrm{MEP}=$ megakaryocyteerythroid progenitor; $\mathrm{PM}=$ promyelocyte; $\mathrm{BC}=$ band cell; $\mathrm{MM}=$ metamyelocyte; $\mathrm{MY}=$ myelocyte; MDS = myelodysplastic syndrome; NAN = not available.

Panel B shows an interactive hierarchical tree summarising the relationship between the samples displayed. Expression level are visualized by size and colour of the nodes, as intuitively indicated by the colour legend. The full name of cell type abbreviations can be obtained by moving the mouse over the individual nodes. Moreover, nodes can be clicked to collapse a branch of the tree.

Panel $\mathrm{C}$ shows a survival plot (Kaplan Meier analysis) based on a high-quality AML dataset from TCGA. MX1 expression levels were dichotomized (above or below median). Other built-in tools allow the removal of cell populations from the graphs, the export of plots as a PDF file and the comparison of paired populations in the default expression plots using the Student's $t$ test.

Figure 2: Immune PRECOG; correlation between immune gene expression levels and survival in haematological malignancies (https://precog.stanford.edu/about.php). Details about available data sets, patient numbers and disease type are provided in panel $\mathrm{A} . \mathrm{AML}=$ acute myeloid leukaemia; B-ALL; B-cell precursor acute lymphoblastic leukaemia; CLL = chronic lymphocytic leukaemia; $\mathrm{BL}=$ Burkitt lymphoma; $\mathrm{DLBCL}=$ diffuse large $\mathrm{B}$-cell lymphoma; $\mathrm{FL}=$ follicular lymphoma; $\mathrm{MM}=$ multiple myeloma.

Panel B summarises the correlation between publicly available immune gene expression levels and overall survival (z-scores). Grey boxes in the heat map denote missing values. The 22 immune cell populations shown here were identified by Newman and co-workers based on the expression of "signature genes" $[41,43]$. Th = follicular helper T cells; Treg = regulatory $T$ 
cells; DCs = dendritic cells; NK = natural killer.

Panel $\mathrm{C}$ shows two-sided $p$ values that were calculated from $z$-scores. Green denotes correlation with better clinical outcome and red indicates correlation with worse clinical outcome. The abundance of immune cell populations was inferred from transcriptomic data sets using a recently developed analytical tool (CIBERSORT; (ㅌell type Identification $\underline{B} y$

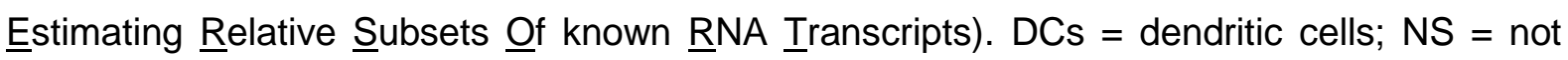
significant.

Figure 3: Approaches to immune biomarker discovery in patients with haematological malignancies. Blood, bone marrow and lymph node samples should be interrogated using genomics and proteomics approaches, immunohistochemistry and flow cytometry to collect comprehensive and personalised profiles on neo-antigen expression, topography and functional orientation of immune cells, tumour specificity of $T$ cells and prognostic immune gene signatures. Lymphoid tissue-resident $T$ cells hold promise as immune effector cells for immunotherapy clinical trials, analogous to the tumour-infiltrating $T$ cells from patients with melanoma [129], in light of recent evidence that ex vivo-expanded marrow-infiltrating lymphocytes (MILs) can be safely administered to patients with high-risk myeloma early after autologous CD34-selected haematopoietic stem cell transplantation [130]. Patients on immunotherapy clinical trials should be sampled sequentially in order to discover and validate mechanistic immune gene signatures associated with response to treatment and/or failure to respond. The gut microbiome could be manipulated to optimise immunotherapeutic responses to checkpoint blockade, as reviewed elsewhere [131].

FFPE = formalin-fixed paraffin-embedded; PBMCs = peripheral blood mononuclear cells; MDSCs = myeloid-derived suppressor cells; TAMs = tumour-associated macrophages; DCs = dendritic cells; MILs = marrow-infiltrating lymphocytes; IHC = immunohistochemistry; B-reg $=$ regulatory $\mathrm{B}$ cells; TCRs $=\mathrm{T}$-cell receptors; Th1 $=\mathrm{T}$-helper type $1 ; \mathrm{IDO} 1=$ indoleamine $2,3-$ dioxygenase-1; ARG2 = arginase 2.

Table 1: On-line resources for the meta-analysis of the prognostic value of immune genes in patients with haematological malignancies. Several platforms are now available for evaluating potential biomarkers (including immune biomarkers) and identifying therapeutic targets in patients with haematological malignancies, including PRECOG [43], PrognoScan [40], BloodSpot [128] and PROGgeneV2 [42]. The above on-line tools and interfaces allow the user to generate Kaplan Meier survival curves and to download gene expression and survival data to be subsequently employed for Cox proportional hazard regression analyses and for robust validation of the biomarker(s) of interest. Most available data sets encompass expression data generated on gene arrays, where individual genes might be represented by 
multiple probe IDs with potentially different specificity for a given RNA sequence. RNA sequencing data do not suffer from this inherent limitation and can be accessed through the TCGA data portal [132]. AML = acute myeloid leukaemia; DLBCL = diffuse large B-cell lymphoma; $\mathrm{FL}$ = follicular lymphoma; RAEB = refractory anaemia with excess of blasts; CLL = chronic lymphocytic leukaemia. 


\section{References}

Papers of special note have been highlighted as either of interest $(\cdot)$ or of considerable interest $(\bullet)$ to readers.

1. Palucka, A.K.; Coussens, L.M. The basis of Oncoimmunology. Cell 2016, 164, 12331247.

2. Blank, C.U.; Haanen, J.B.; Ribas, A.; Schumacher, T.N. The "cancer immunogram". Science 2016, 352, 658-660.

3. Gnjatic, S.; Bronte, V.; Brunet, L.R.; Butler, M.O.; Disis, M.L.; Galon, J.; Hakansson, L.G.; Hanks, B.A.; Karanikas, V.; Khleif, S.N., et al. Identifying baseline immunerelated biomarkers to predict clinical outcome of immunotherapy. $J$ Immunother Cancer 2017, 5, 44.

4. Anderson, K.G.; Stromnes, I.M.; Greenberg, P.D. Obstacles posed by the tumor microenvironment to T cell activity: A case for synergistic therapies. Cancer Cell 2017, 31, 311-325.

5. Chen, D.S.; Mellman, I. Oncology meets immunology: the cancer-immunity cycle. Immunity 2013, 39, 1-10.

6. Iglesia, M.D.; Parker, J.S.; Hoadley, K.A.; Serody, J.S.; Perou, C.M.; Vincent, B.G. Genomic analysis of immune cell infiltrates across 11 tumor types. J Natl Cancer Inst 2016, 108.

7. Galon, J.; Costes, A.; Sanchez-Cabo, F.; Kirilovsky, A.; Mlecnik, B.; Lagorce-Pages, C.; Tosolini, M.; Camus, M.; Berger, A.; Wind, P., et al. Type, density, and location of immune cells within human colorectal tumors predict clinical outcome. Science 2006, 313, 1960-1964.

8. Armand, P.; Shipp, M.A.; Ribrag, V.; Michot, J.M.; Zinzani, P.L.; Kuruvilla, J.; Snyder, E.S.; Ricart, A.D.; Balakumaran, A.; Rose, S., et al. Programmed Death-1 blockade with pembrolizumab in patients with classical Hodgkin lymphoma after brentuximab vedotin failure. J Clin Oncol 2016.

9. $\quad$ Ansell, S.M.; Lesokhin, A.M.; Borrello, I.; Halwani, A.; Scott, E.C.; Gutierrez, M.; Schuster, S.J.; Millenson, M.M.; Cattry, D.; Freeman, G.J., et al. PD-1 blockade with nivolumab in relapsed or refractory Hodgkin's lymphoma. N Engl J Med 2015, 372, 311-319.

10. Melero, I.; Berman, D.M.; Aznar, M.A.; Korman, A.J.; Perez Gracia, J.L.; Haanen, J. Evolving synergistic combinations of targeted immunotherapies to combat cancer. Nat Rev Cancer 2015, 15, 457-472.

11. Stroncek, D.F.; Butterfield, L.H.; Cannarile, M.A.; Dhodapkar, M.V.; Greten, T.F.; Grivel, J.C.; Kaufman, D.R.; Kong, H.H.; Korangy, F.; Lee, P.P., et al. Systematic evaluation of immune regulation and modulation. J Immunother Cancer 2017, 5, 21.

12. Farsaci, B.; Donahue, R.N.; Grenga, I.; Lepone, L.M.; Kim, P.S.; Dempsey, B.; Siebert, J.C.; Ibrahim, N.K.; Madan, R.A.; Heery, C.R., et al. Analyses of pretherapy peripheral immunoscore and response to vaccine therapy. Cancer Immunol Res 2016, 4, 755765.

13. Wargo, J.A.; Reddy, S.M.; Reuben, A.; Sharma, P. Monitoring immune responses in the tumor microenvironment. Curr Opin Immunol 2016, 41, 23-31.

14. Mlecnik, B.; Bindea, G.; Angell, H.K.; Sasso, M.S.; Obenauf, A.C.; Fredriksen, T.; Lafontaine, L.; Bilocq, A.M.; Kirilovsky, A.; Tosolini, M., et al. Functional network pipeline reveals genetic determinants associated with in situ lymphocyte proliferation and survival of cancer patients. Sci Transl Med 2014, 6, 228ra237.

15. Bindea, G.; Mlecnik, B.; Tosolini, M.; Kirilovsky, A.; Waldner, M.; Obenauf, A.C.; Angell, H.; Fredriksen, T.; Lafontaine, L.; Berger, A., et al. Spatiotemporal dynamics of intratumoral immune cells reveal the immune landscape in human cancer. Immunity 2013, 39, 782-795.

16. Pardoll, D.M. The blockade of immune checkpoints in cancer immunotherapy. Nat Rev Cancer 2012, 12, 252-264. 
17. Taube, J.M.; Anders, R.A.; Young, G.D.; Xu, H.; Sharma, R.; McMiller, T.L.; Chen, S.; Klein, A.P.; Pardoll, D.M.; Topalian, S.L., et al. Colocalization of inflammatory response with B7-H1 expression in human melanocytic lesions supports an adaptive resistance mechanism of immune escape. Sci Transl Med 2012, 4, 127 ra137.

18. Teng, M.W.; Ngiow, S.F.; Ribas, A.; Smyth, M.J. Classifying cancers based on T-cell infiltration and PD-L1. Cancer Res 2015, 75, 2139-2145.

19. Parsa, A.T.; Waldron, J.S.; Panner, A.; Crane, C.A.; Parney, I.F.; Barry, J.J.; Cachola, K.E.; Murray, J.C.; Tihan, T.; Jensen, M.C., et al. Loss of tumor suppressor PTEN function increases B7-H1 expression and immunoresistance in glioma. Nat Med 2007, 13, 84-88.

20. Marzec, M.; Zhang, Q.; Goradia, A.; Raghunath, P.N.; Liu, X.; Paessler, M.; Wang, H.Y.; Wysocka, M.; Cheng, M.; Ruggeri, B.A., et al. Oncogenic kinase NPM/ALK induces through STAT3 expression of immunosuppressive protein CD274 (PD-L1, B7H1). Proc Natl Acad Sci U S A 2008, 105, 20852-20857.

21. Chen, D.S.; Mellman, I. Elements of cancer immunity and the cancer-immune set point. Nature 2017, 541, 321-330.

22. Herbst, R.S.; Soria, J.C.; Kowanetz, M.; Fine, G.D.; Hamid, O.; Gordon, M.S.; Sosman, J.A.; McDermott, D.F.; Powderly, J.D.; Gettinger, S.N., et al. Predictive correlates of response to the anti-PD-L1 antibody MPDL3280A in cancer patients. Nature 2014, 515, 563-567.

23. Tumeh, P.C.; Harview, C.L.; Yearley, J.H.; Shintaku, I.P.; Taylor, E.J.; Robert, L.; Chmielowski, B.; Spasic, M.; Henry, G.; Ciobanu, V., et al. PD-1 blockade induces responses by inhibiting adaptive immune resistance. Nature 2014, 515, 568-571.

24. Mercier, F.E.; Ragu, C.; Scadden, D.T. The bone marrow at the crossroads of blood and immunity. Nat Rev Immunol 2011, 12, 49-60.

25. Spitzer, M.H.; Gherardini, P.F.; Fragiadakis, G.K.; Bhattacharya, N.; Yuan, R.T.; Hotson, A.N.; Finck, R.; Carmi, Y.; Zunder, E.R.; Fantl, W.J., et al. An interactive reference framework for modeling a dynamic immune system. Science 2015, 349, 1259425.

26. Curran, E.K.; Godfrey, J.; Kline, J. Mechanisms of immune tolerance in leukemia and lymphoma. Trends Immunol 2017.

27. Mussai, F.; De Santo, C.; Abu-Dayyeh, I.; Booth, S.; Quek, L.; McEwen-Smith, R.M.; Qureshi, A.; Dazzi, F.; Vyas, P.; Cerundolo, V. Acute myeloid leukemia creates an arginase-dependent immunosuppressive microenvironment. Blood 2013, 122, 749758.

28. Folgiero, V.; Goffredo, B.M.; Filippini, P.; Masetti, R.; Bonanno, G.; Caruso, R.; Bertaina, V.; Mastronuzzi, A.; Gaspari, S.; Zecca, M., et al. Indoleamine 2,3dioxygenase 1 (IDO1) activity in leukemia blasts correlates with poor outcome in childhood acute myeloid leukemia. Oncotarget 2014, 5, 2052-2064.

29. Curti, A.; Pandolfi, S.; Valzasina, B.; Aluigi, M.; Isidori, A.; Ferri, E.; Salvestrini, V.; Bonanno, G.; Rutella, S.; Durelli, I., et al. Modulation of tryptophan catabolism by human leukemic cells results in the conversion of CD25- into CD25+ T regulatory cells. Blood 2007, 109, 2871-2877.

30. Chen, B.J.; Chapuy, B.; Ouyang, J.; Sun, H.H.; Roemer, M.G.; Xu, M.L.; Yu, H.; Fletcher, C.D.; Freeman, G.J.; Shipp, M.A., et al. PD-L1 expression is characteristic of a subset of aggressive B-cell lymphomas and virus-associated malignancies. Clin Cancer Res 2013, 19, 3462-3473.

31. Horlad, H.; Ma, C.; Yano, H.; Pan, C.; Ohnishi, K.; Fujiwara, Y.; Endo, S.; Kikukawa, Y.; Okuno, Y.; Matsuoka, M., et al. An IL-27/Stat3 axis induces expression of programmed cell death 1 ligands (PD-L1/2) on infiltrating macrophages in lymphoma. Cancer Sci 2016, 107, 1696-1704.

32. Gibney, G.T.; Weiner, L.M.; Atkins, M.B. Predictive biomarkers for checkpoint inhibitorbased immunotherapy. Lancet Oncol 2016, 17, e542-e551.

33. Ribas, A.; Robert, C.; Hodi, F.S.; Wolchok, J.D.; Joshua, A.M.; Hwu, W.-J.; Weber, J.S.; Zarour, H.M.; Kefford, R.; Loboda, A., et al. Association of response to 
programmed death receptor 1 (PD-1) blockade with pembrolizumab (MK-3475) with an interferon-inflammatory immune gene signature. J Clin Oncol 2015, 33, 3001-3001.

34. Mlecnik, B.; Bindea, G.; Kirilovsky, A.; Angell, H.K.; Obenauf, A.C.; Tosolini, M.; Church, S.E.; Maby, P.; Vasaturo, A.; Angelova, M., et al. The tumor microenvironment and Immunoscore are critical determinants of dissemination to distant metastasis. Sci Transl Med 2016, 8, 327ra326.

35. Chen, P.L.; Roh, W.; Reuben, A.; Cooper, Z.A.; Spencer, C.N.; Prieto, P.A.; Miller, J.P.; Bassett, R.L.; Gopalakrishnan, V.; Wani, K., et al. Analysis of immune signatures in longitudinal tumor samples yields insight into biomarkers of response and mechanisms of resistance to immune checkpoint blockade. Cancer Discov 2016, 6, 827-837.

36. Brown, S.D.; Warren, R.L.; Gibb, E.A.; Martin, S.D.; Spinelli, J.J.; Nelson, B.H.; Holt, R.A. Neo-antigens predicted by tumor genome meta-analysis correlate with increased patient survival. Genome Res 2014, 24, 743-750.

37. Rooney, M.S.; Shukla, S.A.; Wu, C.J.; Getz, G.; Hacohen, N. Molecular and genetic properties of tumors associated with local immune cytolytic activity. Cell 2015, 160, 48-61.

38. Danaher, P.; Warren, S.; Dennis, L.; D'Amico, L.; White, A.; Disis, M.L.; Geller, M.A.; Odunsi, K.; Beechem, J.; Fling, S.P. Gene expression markers of tumor infiltrating leukocytes. J Immunother Cancer 2017, 5, 18.

39. Church, S.E.; Galon, J. Tumor microenvironment and immunotherapy: The whole picture Is better than a glimpse. Immunity 2015, 43, 631-633.

40. Mizuno, H.; Kitada, K.; Nakai, K.; Sarai, A. PrognoScan: a new database for metaanalysis of the prognostic value of genes. BMC Med Genomics 2009, 2, 18.

41. Newman, A.M.; Liu, C.L.; Green, M.R.; Gentles, A.J.; Feng, W.; Xu, Y.; Hoang, C.D.; Diehn, M.; Alizadeh, A.A. Robust enumeration of cell subsets from tissue expression profiles. Nat Methods 2015, 12, 453-457.

42. Goswami, C.P.; Nakshatri, H. PROGgeneV2: enhancements on the existing database. BMC Cancer 2014, 14, 970.

43. Gentles, A.J.; Newman, A.M.; Liu, C.L.; Bratman, S.V.; Feng, W.; Kim, D.; Nair, V.S.; $\mathrm{Xu}, \mathrm{Y}$.; Khuong, A.; Hoang, C.D., et al. The prognostic landscape of genes and infiltrating immune cells across human cancers. Nat Med 2015, 21, 938-945.

44. Austin, R.; Smyth, M.J.; Lane, S.W. Harnessing the immune system in acute myeloid leukaemia. Crit Rev Oncol Hematol 2016, 103, 62-77.

45. Hara, T.; Matsumoto, T.; Shibata, Y.; Nakamura, N.; Nakamura, H.; Ninomiya, S.; Kitagawa, J.; Nannya, Y.; Shimizu, M.; Ito, H., et al. Prognostic value of the combination of serum L-kynurenine level and indoleamine 2,3-dioxygenase mRNA expression in acute myeloid leukemia. Leuk Lymphoma 2016, 57, 2208-2211.

46. Jitschin, R.; Braun, M.; Buttner, M.; Dettmer-Wilde, K.; Bricks, J.; Berger, J.; Eckart, M.J.; Krause, S.W.; Oefner, P.J.; Le Blanc, K., et al. CLL-cells induce IDOhi CD14+HLA-DRlo myeloid-derived suppressor cells that inhibit T-cell responses and promote TRegs. Blood 2014, 124, 750-760.

47. Saulep-Easton, D.; Vincent, F.B.; Le Page, M.; Wei, A.; Ting, S.B.; Croce, C.M.; Tam, C.; Mackay, F. Cytokine-driven loss of plasmacytoid dendritic cell function in chronic lymphocytic leukemia. Leukemia 2014, 28, 2005-2015.

48. Ramsay, A.G.; Clear, A.J.; Fatah, R.; Gribben, J.G. Multiple inhibitory ligands induce impaired T-cell immunologic synapse function in chronic lymphocytic leukemia that can be blocked with lenalidomide: establishing a reversible immune evasion mechanism in human cancer. Blood 2012, 120, 1412-1421.

49. Ding, W.; LaPlant, B.R.; Call, T.G.; Parikh, S.A.; Leis, J.F.; He, R.; Shanafelt, T.D.; Sinha, S.; Le-Rademacher, J.; Feldman, A.L., et al. Pembrolizumab in patients with CLL and Richter transformation or with relapsed CLL. Blood 2017, 129, 3419-3427.

50. Zitvogel, L.; Rusakiewicz, S.; Routy, B.; Ayyoub, M.; Kroemer, G. Immunological offtarget effects of imatinib. Nat Rev Clin Oncol 2016, 13, 431-446. 
51. Gullaksen, S.E.; Skavland, J.; Gavasso, S.; Tosevski, V.; Warzocha, K.; Dumrese, C.; Ferrant, A.; Gedde-Dahl, T.; Hellmann, A.; Janssen, J., et al. Single cell immune profiling by mass cytometry of newly diagnosed chronic phase chronic myeloid leukaemia treated with nilotinib. Haematologica 2017.

52. Ouyang, J.; Plutschow, A.; Pogge von Strandmann, E.; Reiners, K.S.; Ponader, S.; Rabinovich, G.A.; Neuberg, D.; Engert, A.; Shipp, M.A. Galectin-1 serum levels reflect tumor burden and adverse clinical features in classical Hodgkin lymphoma. Blood 2013, 121, 3431-3433.

53. Steidl, C.; Lee, T.; Shah, S.P.; Farinha, P.; Han, G.; Nayar, T.; Delaney, A.; Jones, S.J.; Iqbal, J.; Weisenburger, D.D., et al. Tumor-associated macrophages and survival in classic Hodgkin's lymphoma. N Engl J Med 2010, 362, 875-885.

54. Tan, K.L.; Scott, D.W.; Hong, F.; Kahl, B.S.; Fisher, R.I.; Bartlett, N.L.; Advani, R.H.; Buckstein, R.; Rimsza, L.M.; Connors, J.M., et al. Tumor-associated macrophages predict inferior outcomes in classic Hodgkin lymphoma: a correlative study from the E2496 Intergroup trial. Blood 2012, 120, 3280-3287.

55. Epeldegui, M.; Lee, J.Y.; Martinez, A.C.; Widney, D.P.; Magpantay, L.I.; Regidor, D.; Mitsuyasu, R.; Sparano, J.A.; Ambinder, R.F.; Martinez-Maza, O. Predictive value of cytokines and immune activation biomarkers in AIDS-related non-Hodgkin lymphoma treated with rituximab plus infusional EPOCH (AMC-034 trial). Clin Cancer Res 2016, 22, 328-336.

56. Vendrame, E.; Hussain, S.K.; Breen, E.C.; Magpantay, L.I.; Widney, D.P.; Jacobson, L.P.; Variakojis, D.; Knowlton, E.R.; Bream, J.H.; Ambinder, R.F., et al. Serum levels of cytokines and biomarkers for inflammation and immune activation, and HIVassociated non-Hodgkin B-cell lymphoma risk. Cancer Epidemiol Biomarkers Prev 2014, 23, 343-349.

57. Breen, E.C.; Hussain, S.K.; Magpantay, L.; Jacobson, L.P.; Detels, R.; Rabkin, C.S.; Kaslow, R.A.; Variakojis, D.; Bream, J.H.; Rinaldo, C.R., et al. B-cell stimulatory cytokines and markers of immune activation are elevated several years prior to the diagnosis of systemic AIDS-associated non-Hodgkin B-cell lymphoma. Cancer Epidemiol Biomarkers Prev 2011, 20, 1303-1314.

58. Purdue, M.P.; Hofmann, J.N.; Kemp, T.J.; Chaturvedi, A.K.; Lan, Q.; Park, J.H.; Pfeiffer, R.M.; Hildesheim, A.; Pinto, L.A.; Rothman, N. A prospective study of 67 serum immune and inflammation markers and risk of non-Hodgkin lymphoma. Blood 2013, 122, 951-957.

59. Carvalho, A.; Cunha, C.; Almeida, A.J.; Osorio, N.S.; Saraiva, M.; Teixeira-Coelho, M.; Pedreiro, S.; Torrado, E.; Domingues, N.; Gomes-Alves, A.G., et al. The rs5743836 polymorphism in TLR9 confers a population-based increased risk of non-Hodgkin lymphoma. Genes Immun 2012, 13, 197-201.

60. Hosgood, H.D., 3rd; Purdue, M.P.; Wang, S.S.; Zheng, T.; Morton, L.M.; Lan, Q.; Menashe, I.; Zhang, Y.; Cerhan, J.R.; Grulich, A., et al. A pooled analysis of three studies evaluating genetic variation in innate immunity genes and non-Hodgkin lymphoma risk. Br J Haematol 2011, 152, 721-726.

61. Dave, S.S.; Wright, G.; Tan, B.; Rosenwald, A.; Gascoyne, R.D.; Chan, W.C.; Fisher, R.I.; Braziel, R.M.; Rimsza, L.M.; Grogan, T.M., et al. Prediction of survival in follicular lymphoma based on molecular features of tumor-infiltrating immune cells. $N$ Engl $J$ Med 2004, 351, 2159-2169.

62. Taskinen, M.; Karjalainen-Lindsberg, M.L.; Leppa, S. Prognostic influence of tumorinfiltrating mast cells in patients with follicular lymphoma treated with rituximab and CHOP. Blood 2008, 111, 4664-4667.

63. Alizadeh, A.A.; Eisen, M.B.; Davis, R.E.; Ma, C.; Lossos, I.S.; Rosenwald, A.; Boldrick, J.C.; Sabet, H.; Tran, T.; Yu, X., et al. Distinct types of diffuse large B-cell lymphoma identified by gene expression profiling. Nature 2000, 403, 503-511.

64. Xing, W.; Dresser, K.; Zhang, R.; Evens, A.M.; Yu, H.; Woda, B.A.; Chen, B.J. PD-L1 expression in EBV-negative diffuse large B-cell lymphoma: clinicopathologic features and prognostic implications. Oncotarget 2016, 7, 59976-59986. 
65. Riihijarvi, S.; Fiskvik, I.; Taskinen, M.; Vajavaara, H.; Tikkala, M.; Yri, O.; KarjalainenLindsberg, M.L.; Delabie, J.; Smeland, E.; Holte, H., et al. Prognostic influence of macrophages in patients with diffuse large B-cell lymphoma: a correlative study from a Nordic phase II trial. Haematologica 2015, 100, 238-245.

66. Gomez-Gelvez, J.C.; Salama, M.E.; Perkins, S.L.; Leavitt, M.; Inamdar, K.V. Prognostic impact of tumor microenvironment in diffuse large B-cell lymphoma uniformly treated with R-CHOP chemotherapy. Am J Clin Pathol 2016, 145, 514-523.

67. Keane, C.; Vari, F.; Hertzberg, M.; Cao, K.A.; Green, M.R.; Han, E.; Seymour, J.F.; Hicks, R.J.; Gill, D.; Crooks, P., et al. Ratios of T-cell immune effectors and checkpoint molecules as prognostic biomarkers in diffuse large B-cell lymphoma: a populationbased study. Lancet Haematol 2015, 2, e445-455.

68. Martelli, M.; Ferreri, A.; Di Rocco, A.; Ansuinelli, M.; Johnson, P.W.M. Primary mediastinal large B-cell lymphoma. Crit Rev Oncol Hematol 2017, 113, 318-327.

69. Mottok, A.; Woolcock, B.; Chan, F.C.; Tong, K.M.; Chong, L.; Farinha, P.; Telenius, A.; Chavez, E.; Ramchandani, S.; Drake, M., et al. Genomic alterations in CIITA are frequent in primary mediastinal large $B$ cell lymphoma and are associated with diminished MHC class II expression. Cell Rep 2015, 13, 1418-1431.

70. Shi, M.; Roemer, M.G.; Chapuy, B.; Liao, X.; Sun, H.; Pinkus, G.S.; Shipp, M.A.; Freeman, G.J.; Rodig, S.J. Expression of programmed cell death 1 ligand 2 (PD-L2) is a distinguishing feature of primary mediastinal (thymic) large B-cell lymphoma and associated with PDCD1LG2 copy gain. Am J Surg Pathol 2014, 38, 1715-1723.

71. Zinzani, P.L.; Ribrag, V.; Moskowitz, C.H.; Michot, J.M.; Kuruvilla, J.; Balakumaran, A.; Zhang, Y.; Chlosta, S.; Shipp, M.A.; Armand, P. Safety and tolerability of pembrolizumab in patients with relapsed/refractory primary mediastinal large B-cell lymphoma. Blood 2017, 130, 267-270.

72. Steidl, C.; Gascoyne, R.D. The molecular pathogenesis of primary mediastinal large B-cell lymphoma. Blood 2011, 118, 2659-2669.

73. Wang, H.; Wang, L.; Chi, P.D.; Wang, W.D.; Chen, X.Q.; Geng, Q.R.; Xia, Z.J.; Lu, Y. High level of interleukin-10 in serum predicts poor prognosis in multiple myeloma. $\mathrm{Br}$ J Cancer 2016, 114, 463-468.

74. Gorgun, G.T.; Whitehill, G.; Anderson, J.L.; Hideshima, T.; Maguire, C.; Laubach, J.; Raje, N.; Munshi, N.C.; Richardson, P.G.; Anderson, K.C. Tumor-promoting immunesuppressive myeloid-derived suppressor cells in the multiple myeloma microenvironment in humans. Blood 2013, 121, 2975-2987.

75. Prabhala, R.H.; Pelluru, D.; Fulciniti, M.; Prabhala, H.K.; Nanjappa, P.; Song, W.; Pai, C.; Amin, S.; Tai, Y.T.; Richardson, P.G., et al. Elevated IL-17 produced by TH17 cells promotes myeloma cell growth and inhibits immune function in multiple myeloma. Blood 2010, 115, 5385-5392.

76. Atanackovic, D.; Luetkens, T.; Kroger, N. Coinhibitory molecule PD-1 as a potential target for the immunotherapy of multiple myeloma. Leukemia 2014, 28, 993-1000.

77. Gorgun, G.; Samur, M.K.; Cowens, K.B.; Paula, S.; Bianchi, G.; Anderson, J.E.; White, R.E.; Singh, A.; Ohguchi, H.; Suzuki, R., et al. Lenalidomide enhances immune checkpoint blockade-induced immune response in multiple myeloma. Clin Cancer Res 2015, 21, 4607-4618.

78. Bonanno, G.; Mariotti, A.; Procoli, A.; Folgiero, V.; Natale, D.; De Rosa, L.; Majolino, I.; Novarese, L.; Rocci, A.; Gambella, M., et al. Indoleamine 2,3-dioxygenase 1 (IDO1) activity correlates with immune system abnormalities in multiple myeloma. $J$ Trans $/$ Med 2012, 10, 247.

79. de Haart, S.J.; van de Donk, N.W.; Minnema, M.C.; Huang, J.H.; Aarts-Riemens, T.; Bovenschen, N.; Yuan, H.; Groen, R.W.; McMillin, D.W.; Jakubikova, J., et al. Accessory cells of the microenvironment protect multiple myeloma from T-cell cytotoxicity through cell adhesion-mediated immune resistance. Clin Cancer Res 2013, 19, 5591-5601.

80. Paiva, B.; Mateos, M.V.; Sanchez-Abarca, L.I.; Puig, N.; Vidriales, M.B.; Lopez-Corral, L.; Corchete, L.A.; Hernandez, M.T.; Bargay, J.; de Arriba, F., et al. Immune status of 
high-risk smoldering multiple myeloma patients and its therapeutic modulation under LenDex: a longitudinal analysis. Blood 2016, 127, 1151-1162.

81. Brown, R.; Kabani, K.; Favaloro, J.; Yang, S.; Ho, P.J.; Gibson, J.; Fromm, P.; Suen, H.; Woodland, N.; Nassif, N., et al. CD86+ or HLA-G+ can be transferred via trogocytosis from myeloma cells to $\mathrm{T}$ cells and are associated with poor prognosis. Blood 2012, 120, 2055-2063.

82. Maude, S.L.; Frey, N.; Shaw, P.A.; Aplenc, R.; Barrett, D.M.; Bunin, N.J.; Chew, A.; Gonzalez, V.E.; Zheng, Z.; Lacey, S.F., et al. Chimeric antigen receptor T cells for sustained remissions in leukemia. N Engl J Med 2014, 371, 1507-1517.

83. Kochenderfer, J.N.; Somerville, R.P.T.; Lu, T.; Shi, V.; Bot, A.; Rossi, J.; Xue, A.; Goff, S.L.; Yang, J.C.; Sherry, R.M., et al. Lymphoma remissions caused by anti-CD19 chimeric antigen receptor $T$ cells are associated with high serum interleukin-15 levels. J Clin Oncol 2017, 35, 1803-1813.

84. Ramos, C.A.; Ballard, B.; Zhang, H.; Dakhova, O.; Gee, A.P.; Mei, Z.; Bilgi, M.; Wu, M.F.; Liu, H.; Grilley, B., et al. Clinical and immunological responses after CD30specific chimeric antigen receptor-redirected lymphocytes. J Clin Invest 2017, 127, 3462-3471.

85. Chong, E.A.; Melenhorst, J.J.; Lacey, S.F.; Ambrose, D.E.; Gonzalez, V.; Levine, B.L.; June, C.H.; Schuster, S.J. PD-1 blockade modulates chimeric antigen receptor (CAR)modified T cells: refueling the CAR. Blood 2017, 129, 1039-1041.

86. Ninomiya, S.; Narala, N.; Huye, L.; Yagyu, S.; Savoldo, B.; Dotti, G.; Heslop, H.E.; Brenner, M.K.; Rooney, C.M.; Ramos, C.A. Tumor indoleamine 2,3-dioxygenase (IDO) inhibits CD19-CAR T cells and is downregulated by lymphodepleting drugs. Blood 2015, 125, 3905-3916.

87. Gomes-Silva, D.; Srinivasan, M.; Sharma, S.; Lee, C.M.; Wagner, D.L.; Davis, T.H.; Rouce, R.H.; Bao, G.; Brenner, M.K.; Mamonkin, M. CD7-edited T cells expressing a CD7-specific CAR for the therapy of T-cell malignancies. Blood 2017, 130, 285-296.

88. Ruella, M.; Barrett, D.M.; Kenderian, S.S.; Shestova, O.; Hofmann, T.J.; Perazzelli, J.; Klichinsky, M.; Aikawa, V.; Nazimuddin, F.; Kozlowski, M., et al. Dual CD19 and CD123 targeting prevents antigen-loss relapses after CD19-directed immunotherapies. J Clin Invest 2016, 126, 3814-3826.

89. Ali, S.A.; Shi, V.; Maric, I.; Wang, M.; Stroncek, D.F.; Rose, J.J.; Brudno, J.N.; StetlerStevenson, M.; Feldman, S.A.; Hansen, B.G., et al. T cells expressing an anti-B-cell maturation antigen chimeric antigen receptor cause remissions of multiple myeloma. Blood 2016, 128, 1688-1700.

90. Garfall, A.L.; Maus, M.V.; Hwang, W.T.; Lacey, S.F.; Mahnke, Y.D.; Melenhorst, J.J.; Zheng, Z.; Vogl, D.T.; Cohen, A.D.; Weiss, B.M., et al. Chimeric antigen receptor T cells against CD19 for multiple myeloma. N Engl J Med 2015, 373, 1040-1047.

91. Ramos, C.A.; Savoldo, B.; Torrano, V.; Ballard, B.; Zhang, H.; Dakhova, O.; Liu, E.; Carrum, G.; Kamble, R.T.; Gee, A.P., et al. Clinical responses with T lymphocytes targeting malignancy-associated kappa light chains. J Clin Invest 2016, 126, 25882596.

92. Kantarjian, H.; Stein, A.; Gokbuget, N.; Fielding, A.K.; Schuh, A.C.; Ribera, J.M.; Wei, A.; Dombret, H.; Foa, R.; Bassan, R., et al. Blinatumomab versus chemotherapy for advanced acute lymphoblastic leukemia. N Engl J Med 2017, 376, 836-847.

93. Bertaina, A.; Filippini, P.; Bertaina, V.; Lucarelli, B.; Bauquet, A.; Zugmaier, G.; Locatelli, F.; Rutella, S. Immune cell phenotype and function after treatment with blinatumomab for childhood relapsed B-cell precursor acute lymphoblastic leukemia (BCP-ALL). Blood 2013, 122, 2668-2668.

94. Feucht, J.; Kayser, S.; Gorodezki, D.; Hamieh, M.; Doring, M.; Blaeschke, F.; Schlegel, P.; Bosmuller, H.; Quintanilla-Fend, L.; Ebinger, M., et al. T-cell responses against CD19+ pediatric acute lymphoblastic leukemia mediated by bispecific T-cell engager (BiTE) are regulated contrarily by PD-L1 and CD80/CD86 on leukemic blasts. Oncotarget 2016, 7, 76902-76919. 
95. Mahoney, K.M.; Rennert, P.D.; Freeman, G.J. Combination cancer immunotherapy and new immunomodulatory targets. Nat Rev Drug Discov 2015, 14, 561-584.

96. Stack, E.C.; Foukas, P.G.; Lee, P.P. Multiplexed tissue biomarker imaging. J Immunother Cancer 2016, 4, 9.

97. Chang, A.Y.; Bhattacharya, N.; Mu, J.; Setiadi, A.F.; Carcamo-Cavazos, V.; Lee, G.H.; Simons, D.L.; Yadegarynia, S.; Hemati, K.; Kapelner, A., et al. Spatial organization of dendritic cells within tumor draining lymph nodes impacts clinical outcome in breast cancer patients. J Transl Med 2013, 11, 242.

98. Cesano, A. nCounter(R) PanCancer Immune Profiling Panel (NanoString Technologies, Inc., Seattle, WA). J Immunother Cancer 2015, 3, 42.

99. Miotto, R.; Wang, F.; Wang, S.; Jiang, X.; Dudley, J.T. Deep learning for healthcare: review, opportunities and challenges. Brief Bioinform 2017.

100. Chatzimichail, E.; Matthaios, D.; Bouros, D.; Karakitsos, P.; Romanidis, K.; Kakolyris, S.; Papashinopoulos, G.; Rigas, A. gamma-H2AX: A novel prognostic marker in a prognosis prediction model of patients with early operable non-small cell lung cancer. Int J Genomics 2014, 2014, 160236.

101. Bertolaccini, L.; Solli, P.; Pardolesi, A.; Pasini, A. An overview of the use of artificial neural networks in lung cancer research. J Thorac Dis 2017, 9, 924-931.

102. Abdel-Fatah, T.M.; Agarwal, D.; Liu, D.X.; Russell, R.; Rueda, O.M.; Liu, K.; Xu, B.; Moseley, P.M.; Green, A.R.; Pockley, A.G., et al. SPAG5 as a prognostic biomarker and chemotherapy sensitivity predictor in breast cancer: a retrospective, integrated genomic, transcriptomic, and protein analysis. Lancet Oncol 2016, 17, 1004-1018.

103. Mehdy, M.M.; Ng, P.Y.; Shair, E.F.; Saleh, N.I.M.; Gomes, C. Artificial neural networks in image processing for early detection of breast cancer. Comput Math Methods Med 2017, 2017, 2610628.

104. Huang, S.H.; Loh, J.K.; Tsai, J.T.; Houg, M.F.; Shi, H.Y. Predictive model for 5-year mortality after breast cancer surgery in Taiwan residents. Chin J Cancer 2017, 36, 23.

105. Abdel-Fatah, T.M.; McArdle, S.E.; Agarwal, D.; Moseley, P.M.; Green, A.R.; Ball, G.R.; Pockley, A.G.; Ellis, I.O.; Rees, R.C.; Chan, S.Y. HAGE in triple-negative breast cancer is a novel prognostic, predictive, and actionable biomarker: A transcriptomic and protein expression analysis. Clin Cancer Res 2016, 22, 905-914.

106. Haj-Hassan, H.; Chaddad, A.; Harkouss, Y.; Desrosiers, C.; Toews, M.; Tanougast, C. Classifications of multispectral colorectal cancer tissues using convolution neural network. J Pathol Inform 2017, 8, 1.

107. Makris, G.M.; Pouliakis, A.; Siristatidis, C.; Margari, N.; Terzakis, E.; Koureas, N.; Pergialiotis, V.; Papantoniou, N.; Karakitsos, P. Image analysis and multi-layer perceptron artificial neural networks for the discrimination between benign and malignant endometrial lesions. Diagn Cytopathol 2017, 45, 202-211.

108. Van Belle, V.; Van Calster, B.; Van Huffel, S.; Suykens, J.A.; Lisboa, P. Explaining support vector machines: A color based nomogram. PLoS One 2016, 11, e0164568.

109. Powell, R.T.; Olar, A.; Narang, S.; Rao, G.; Sulman, E.; Fuller, G.N.; Rao, A. Identification of histological correlates of overall survival in lower grade gliomas using a bag-of-words paradigm: A preliminary analysis based on hematoxylin \& eosin stained slides from the lower grade glioma cohort of the Cancer Genome Atlas. $J$ Pathol Inform 2017, 8, 9.

110. Araujo, T.; Aresta, G.; Castro, E.; Rouco, J.; Aguiar, P.; Eloy, C.; Polonia, A.; Campilho, A. Classification of breast cancer histology images using Convolutional Neural Networks. PLoS One 2017, 12, e0177544.

111. Huang, M.W.; Chen, C.W.; Lin, W.C.; Ke, S.W.; Tsai, C.F. SVM and SVM ensembles in breast cancer prediction. PLoS One 2017, 12, e0161501.

112. Cheerla, N.; Gevaert, O. MicroRNA based pan-cancer diagnosis and treatment recommendation. BMC Bioinformatics 2017, 18, 32.

113. Zeng, Z.; Jiang, X.; Neapolitan, R. Discovering causal interactions using Bayesian network scoring and information gain. BMC Bioinformatics 2016, 17, 221. 
114. Zhu, X.; Ko, Y.J.; Berry, S.; Shah, K.; Lee, E.; Chan, K. A Bayesian network metaanalysis on second-line systemic therapy in advanced gastric cancer. Gastric Cancer 2017, 20, 646-654.

115. Field, S.L.; Dasgupta, T.; Cummings, M.; Savage, R.S.; Adebayo, J.; McSara, H.; Gunawardena, J.; Orsi, N.M. Bayesian modeling suggests that IL-12 (p40), IL-13 and MCP-1 drive murine cytokine networks in vivo. BMC Syst Biol 2015, 9, 76.

116. Luo, Y.; El Naqa, I.; McShan, D.L.; Ray, D.; Lohse, I.; Matuszak, M.M.; Owen, D.; Jolly, S.; Lawrence, T.S.; Kong, F.S., et al. Unraveling biophysical interactions of radiation pneumonitis in non-small-cell lung cancer via Bayesian network analysis. Radiother Oncol 2017, 123, 85-92.

117. Sommer, C.; Gerlich, D.W. Machine learning in cell biology - teaching computers to recognize phenotypes. J Cell Sci 2013, 126, 5529-5539.

118. Stadler, N.; Dondelinger, F.; Hill, S.M.; Akbani, R.; Lu, Y.; Mills, G.B.; Mukherjee, S. Molecular heterogeneity at the network level: high-dimensional testing, clustering and a TCGA case study. Bioinformatics 2017.

119. Athreya, A.P.; Kalari, K.R.; Cairns, J.; Gaglio, A.J.; Wills, Q.F.; Niu, N.; Weinshilboum, R.; lyer, R.K.; Wang, L. Model-based unsupervised learning informs metformininduced cell-migration inhibition through an AMPK-independent mechanism in breast cancer. Oncotarget 2017, 8, 27199-27215.

120. Vural, S.; Wang, X.; Guda, C. Classification of breast cancer patients using somatic mutation profiles and machine learning approaches. BMC Syst Biol 2016, 10 Suppl 3, 62.

121. Rodriguez, A.; Laio, A. Machine learning. Clustering by fast search and find of density peaks. Science 2014, 344, 1492-1496.

122. Xanthopoulos, P.; Pardalos, P.M.; Trafalis, T.B. Principal Component Analysis. In Robust Data Mining, Springer New York: New York, NY, 2013; pp 21-26.

123. Krzywinski, M.; Birol, I.; Jones, S.J.; Marra, M.A. Hive plots--rational approach to visualizing networks. Brief Bioinform 2012, 13, 627-644.

124. Galon, J.; Angell, H.K.; Bedognetti, D.; Marincola, F.M. The continuum of cancer immunosurveillance: prognostic, predictive, and mechanistic signatures. Immunity 2013, 39, 11-26.

125. Topalian, S.L.; Taube, J.M.; Anders, R.A.; Pardoll, D.M. Mechanism-driven biomarkers to guide immune checkpoint blockade in cancer therapy. Nat Rev Cancer 2016, 16, 275-287.

126. Beatty, G.L.; O'Dwyer, P.J.; Clark, J.; Shi, J.G.; Bowman, K.J.; Scherle, P.A.; Newton, R.C.; Schaub, R.; Maleski, J.; Leopold, L., et al. First-in-human phase I study of the oral inhibitor of indoleamine 2,3-dioxygenase-1 epacadostat (INCB024360) in patients with advanced solid malignancies. Clin Cancer Res 2017.

127. Lesokhin, A.M.; Callahan, M.K.; Postow, M.A.; Wolchok, J.D. On being less tolerant: enhanced cancer immunosurveillance enabled by targeting checkpoints and agonists of T cell activation. Sci Transl Med 2015, 7, 280 sr281.

128. Bagger, F.O.; Sasivarevic, D.; Sohi, S.H.; Laursen, L.G.; Pundhir, S.; Sonderby, C.K.; Winther, O.; Rapin, N.; Porse, B.T. BloodSpot: a database of gene expression profiles and transcriptional programs for healthy and malignant haematopoiesis. Nucleic Acids Res 2016, 44, D917-924.

129. Goff, S.L.; Dudley, M.E.; Citrin, D.E.; Somerville, R.P.; Wunderlich, J.R.; Danforth, D.N.; Zlott, D.A.; Yang, J.C.; Sherry, R.M.; Kammula, U.S., et al. Randomized, prospective evaluation comparing intensity of lymphodepletion before adoptive transfer of tumor-infiltrating lymphocytes for patients with metastatic melanoma. J Clin Oncol 2016, 34, 2389-2397.

130. Noonan, K.A.; Huff, C.A.; Davis, J.; Lemas, M.V.; Fiorino, S.; Bitzan, J.; Ferguson, A.; Emerling, A.; Luznik, L.; Matsui, W., et al. Adoptive transfer of activated marrowinfiltrating lymphocytes induces measurable antitumor immunity in the bone marrow in multiple myeloma. Sci Transl Med 2015, 7, 288ra278. 
131. Roy, S.; Trinchieri, G. Microbiota: a key orchestrator of cancer therapy. Nat Rev Cancer 2017, 17, 271-285.

132. Ley, T.J.; Miller, C.; Ding, L.; Raphael, B.J.; Mungall, A.J.; Robertson, A.; Hoadley, K.; Triche, T.J., Jr.; Laird, P.W.; Baty, J.D., et al. Genomic and epigenomic landscapes of adult de novo acute myeloid leukemia. N Engl J Med 2013, 368, 2059-2074. 